\title{
La representación metafórica del tiempo en la poesía de Yolanda Westphalen
}

\section{The metaphorical representation of time in the poetry of Yolanda Westphalen}

\author{
Jaime Cabrera Junco \\ Universidad Nacional Mayor de San Marcos, Lima, Perú \\ jaime.cabrera@unmsm.edu.pe \\ ORCID: https://orcid.org/0000-0001-9450-9091
}

\section{Resumen}

El siguiente artículo analiza la representación metafórica del tiempo en el poemario Viviendo el tiempo (2008), de Yolanda Westphalen (1920-2011), autora adscrita a la generación del 60. Para este análisis nos apoyaremos en el estudio de Lakoff y Johnson, quienes en Metáforas de la vida cotidiana consideran que el lenguaje es una representación de la experiencia cotidiana. Lo postulado por el investigador italiano Stefano Arduini en su obra Prolegómenos para una teoría general de las figuras nos ofrecerá la fundamentación teórica sobre la construcción de la metáfora del tiempo en algunos poemas representativos seleccionados para este fin.

Palabras clave Poesía, poética, tiempo, metáfora, vivencia

\begin{abstract}
This article analyzes the metaphorical representation of the time in the poetry book Viviendo el tiempo (2008), by Yolanda Westphalen (1920-2011), an author is assigned to the generation of the 60s. For this analysis, we will rely on the study of Lakoff and Johnson, who in Metaphors We Live By consider that the language is a representation of everyday experience. The postulated by the Italian researcher Stefano Arduini in his work Prolegomena for a general theory of figures will provide us a theoretical support on the construction of the metaphor of time in some representative poems selected for this purpose.
\end{abstract}

Keywords Poetry, poetics, time, metaphor, experience

Fecha de envío: 9/3/2021 Fecha de aceptación: 30/5/2021 


\section{Introducción}

Yolanda Rodríguez Cartland de Westphalen (1920-2011) fue una autora bisagra entre las generaciones del 50 y el 60. Por edad podría ser considerada parte de la primera, pero se la incluye en esta última debido a que su primer poemario, Palabra fugitiva, fue publicado en 1964. Su producción abarca fundamentalmente la poesía, aunque también escribió cuentos y artículos recogidos en la prensa escrita y en revistas literarias. Adoptó el apellido Westphalen de su esposo Luis, quien a su vez fue hermano del reconocido poeta Emilio Adolfo Westphalen (19112001). Su formación humanística la realizó en la Facultad de Letras de la Universidad Nacional Mayor de San Marcos, y se especializó en Filosofía. Aunque resulte un dato menor, la poeta inició sus estudios con más de 40 años de edad, y logró incluso obtener el grado de doctora, algo poco usual para las mujeres de aquella época.

Así recuerda Westphalen su primer acercamiento a la poesía:

Desde muy joven amé la poesía y en mi juventud comencé a transcribir en verso el asombro y la emoción que el mundo producía en mí; amaba la sonoridad de la palabra, amaba el condensar todo un juicio racional en la novedad de la metáfora y el símbolo creando la viviente expresión de una nueva dimensión de la palabra, hecha concreción de imagen, sentido y ritmo (Westphalen, 2018b, p. 122).

Para la autora, su paso por las aulas sanmarquinas fue decisivo. Desde su formación humanística y reflexiva, se cimentarán las bases de su obra poética, donde el lirismo se conjuga con la mirada de lo que acontece a su alrededor.

El sueño se hizo realidad, postulé a la Universidad Nacional Mayor de San Marcos y logré ingresar. Estudiando los dos años de Cultura, dos exigencias nacieron en mí: la de la reflexión filosófica y la emoción lírica, todos creen que estos dos mundos son antagónicos, pero para mí no lo eran, la reflexión se adentraba en la profundidad del pensar y la literatura me facultaba para expresar en emoción toda la experiencia del vivir tanto real como oníricamente (p. 122). 
Habiendo publicado ya tres poemarios recién será considerada en una antología poética. Ocurrió en 1984, en un trabajo realizado por el crítico Ricardo González Vigil $1^{1}$, quien fue el primero en ponderar su trabajo. Pasaron poco más de 10 años para que otra vez sea considerada en una selección poética, esta vez realizada por la crítica literaria Esther Castañeda, en 1995. Posteriormente, su obra fue analizada a través de artículos de difusión en el ámbito académico. Su hija e investigadora literaria, Yolanda Westphalen Rodríguez, sostiene que fue "invisibilizada como muchas otras mujeres intelectuales por el canon académico tradicional” (Westphalen, 2018a, p. 13). Por su parte, Castañeda (1995) considera que, junto con Blanca Varela, Westphalen es una autora que inicia tendencias "en pos de una autonomía poética" (p. VI); destaca su indagación reflexiva y personal a partir de dos ejes: el tiempo y las limitaciones de la palabra para expresar las sensaciones del yo poético. Aunque esté más cercana a la generación del 50, la insularidad de su obra la mantuvo al margen de este grupo, sobre todo cuando surgió el debate sobre la llamada "poesía pura” y la "poesía social". Pese a que fue compañera de estudios de algunos integrantes de la llamada generación del 60 - César Calvo, Antonio Cisneros, Reynaldo Naranjo y Arturo Corcuera-, no compartió con ellos intereses temáticos. Para el investigador francés Roland Forgues (2004), la poética de Yolanda Westphalen se distingue por su búsqueda filosófica "una meditación sobre el origen del ser y la existencia enmarcada en los estrechos límites del tiempo y del espacio" (p. 91).

Esta situación de marginalidad cambió a partir de 2018, cuando el Fondo Editorial de la Universidad Nacional Mayor de San Marcos publicó en dos tomos la obra completa de Yolanda Westphalen. El primer volumen está dedicado a su poesía e incluye los nueve poemarios que publicó en vida entre 1964 y 2008, así como un décimo libro inédito titulado Epilogo en dolor escrito. Según su hija y editora, los poemas que conforman esta obra póstuma fueron trabajados aproximadamente en 1992.

Daremos a conocer, entonces, algunas de las características de la obra poética de Yolanda Westphalen. Realizaremos una valoración a partir del tópico principal que atraviesa gran parte de ella: el tiempo como punto de reflexión del yo poético.

\section{Desarrollo}

\section{La poesía de Yolanda Westphalen}

En su ensayo "La poesía peruana en los años sesenta" (2019), el poeta Eduardo Chirinos señala que las características coincidentes en la obra de los autores de 
la generación del 60 son: la versificación libre, el lenguaje conversacional, la aceptación de la imagen como elemento constitutivo del poema bajo las influencias de Ezra Pound y Ernesto Cardenal (Chirinos, 2019, p. 269), así como su heterodoxia política. La instauración de la Revolución cubana y sus ideales políticos influyeron también en el quehacer de los jóvenes poetas de entonces. Esto se evidencia, por ejemplo, en los poemarios Comentarios reales (1964) de Antonio Cisneros y Consejero del lobo (1965) de Rodolfo Hinostroza. Además, resalta la participación activa en los movimientos guerrilleros. El caso más visible es el de Javier Heraud, muerto en Madre de Dios en 1963.

En Antología de la poesía peruana joven (1965), el poeta Francisco Carrillo determina que la llamada generación del 60 buscó acercarse a la realidad cotidiana adoptando un lenguaje coloquial. Esto no significaba, afirma, un desinterés por las “introspecciones humanas y estéticas” (p. 5). En la antología Los nuevos (1967), considerada por la crítica como la que dio oficialmente a conocer a la generación del 60, Leonidas Cevallos considera que "un cambio en la realidad trae un cambio en la poesía” (p. 9). Esta inquietud se traduce en las obras de los jóvenes poetas, las cuales tratan de ajustarlas "a la nueva situación y a los problemas que suscita esta realidad" (p. 9) y buscando dar "testimonio de la experiencia personal" (p. 10).

La obra poética de Yolanda Westphalen se distingue de la generación del 60 al no adoptar el conversacionalismo como propuesta estética ni estar bajo el influjo de la poesía anglosajona. La investigadora Bethsabé Huamán Andía sostiene que, poéticamente, Westphalen se encuentra más cercana a la generación del 50 "en cuanto al purismo de su poesía" (Westphalen, 2018a, p. 40). Ya desde su primer poemario, Palabra fugitiva (1964), la autora evidencia la reflexión que le suscita al yo poético la mirada de lo cotidiano respecto a sus vivencias internas. En Moradas de la voz (2002), el crítico Miguel Ángel Zapata encuentra semejanzas entre la poesía de Westphalen y el simbolismo de José María Eguren. Sin embargo, afirma que "la diferencia radicaría en la reducción del color a la forma de los objetos y la ausencia de todo onirismo" (p. 208). La poeta Marita Troiano (2008) destaca su "acendrada vocación filosófica y lo canónico de sus versos que traducen con sabiduría cotidiana aquel delirio interior que la sume constante en la bruma metafísica" (Troiano, 2008, párr. 1).

El estilo de su poesía es intimista y refleja experiencias como el asombro, el dolor, la ilusión y el fracaso. Tal como indica la investigadora Yolanda Westphalen Rodríguez, la palabra y el tiempo son los ejes temáticos estructurales de toda su obra. Su poesía es, en ese sentido, "vitalista y existencial que vive y se desgarra intensamente, siente, cuestiona y se piensa a sí misma" (Westphalen, 2018a, p. 
15). Su poética es tributaria del modernismo y del simbolismo, pero se distingue en que "la musicalidad y la fantasía, el sonido y el ritmo, adquieren voz propia y subvierten la poética de sus predecesores”(Westphalen, 2018a, p. 19).

Los objetos de la vida diaria sirven de punto de reflexión de la existencia, donde la visión intimista del yo poético destaca sobre la rima y la musicalidad del verso. Es cierto, sin embargo, que en Diptico (1996), la musicalidad se aprecia en la sección "Fuegos fatuos": "Eres tú la voz mutilada / de la lluvia / el perfil alucinante de mis sueños” (Westphalen, 2018, p. 183). Una línea común en toda su poética, sostiene Westphalen Rodríguez, es la relación entre la palabra y el tiempo, a la que le corresponden recursos expresivos "que se caracterizan por el uso permanente de anáforas y metáforas” (Westphalen, 2018a, p. 18).

Esta propuesta poética se evidencia mejor en su último poemario titulado Viviendo el tiempo (2008), donde para la autora "la poesía no solo vive y captura el tiempo, sino que lo trasciende" (Westphalen, 2018a, p.27). Como remarca Huamán Andía, "en sus poemarios últimos logra la perfecta concisión y profundidad que anhelaba” (Westphalen, 2018a, p. 37).

Es a partir de estas afirmaciones que analizaremos cinco poemas representativos de este último libro, donde Westphalen reflexiona sobre la relación entre la vida y el tiempo. En dicho análisis mostraremos cómo aparece representado el tiempo y cómo se realiza su metaforización en el poema, lo cual permite al sujeto lírico expresar un sentimiento existencial más profundo. Antes de esto presentaremos la fundamentación teórica para nuestro análisis.

\section{Estudios recientes sobre su obra}

La reedición de su obra completa suscitó algunos análisis de su poesía, más precisamente a través de artículos publicados en 2020 en la revista Metáfora. En el volumen 3, número 5 , de dicha publicación encontramos dos análisis sobre dos obras de nuestra autora: Objetos enajenados (1971) y el poema "6", de Universo en exilio (1984).

En el primer caso, Christian Cachay (2020) parte del estudio de la retórica de Arduini y las técnicas argumentativas de Chaïm Perelman y Lucie Olbrechts-Tyteca para analizar el segundo poemario de Westphalen. El investigador afirma que en dicha obra la autora parte de una observación crítica de la realidad, así como del diálogo y la personalización, para ofrecernos una visión humanizadora de los objetos cotidianos. En su análisis concluye: 
En Objetos enajenados (1971), la reflexión humana a través de los objetos exteriores, inscritos en un hogar y en la época moderna, tienen como conclusión demostrar la deshumanización del sujeto, su pérdida de valores y la esperanza. En el caso de "el hombre-presa", el perjuicio de la libertad ha cosificado al hombre. Por otro lado, en "el reloj", la condición finita del ser humano es inevitable y su recuerdo, mediante el objeto, desespera al locutor, esto provoca que el poema concluya con el fin de los tiempos demostrando el sinsentido de la existencia (p. 21).

También remarca que la obra de Westphalen todavía se encuentra en pleno proceso de revaloración, precisamente a partir de la publicación de sus obras completas. Asimismo, debido a que su obra acaba de ser publicada de manera total es posible que más adelante se realicen investigaciones más exhaustivas sobre la poeta.

De otro lado, Gaby Salvador (2020) realizó un análisis retórico del poema “6”, incluido en Universo en exilio (1984), el tercer poemario de Westphalen. Dicho poema evidencia una crítica a las posibilidades del lenguaje desde un aspecto filosófico, algo que, según manifiesta Salvador, ha sido invisibilizado por la crítica:

Yolanda Westphalen manifiesta las dudas o contradicciones respecto del lenguaje en función de la capacidad de comunicación y de expresión genuina de lo que pensamos. Además, resaltamos que la crítica ha visibilizado (mas no de manera suficiente) la problemática filosófica de la obra de Westphalen, específicamente en lo que al lenguaje se refiere. Para realizar nuestro análisis, nos hemos valido de los postulados de Stefano Arduini en torno a las nociones de campo retórico y campo figurativo (pp. 11-12).

Esta impronta filosófica y reflexiva sobre el entorno y el tiempo nos llevó a centrarnos en uno de sus poemarios más sólidos y, así, analizar cómo opera la metaforización del tiempo.

\section{La metaforización del tiempo y la mirada cotidiana}

La metaforización del tiempo en Viviendo el tiempo (2008), de Yolanda Westphalen, se construye a partir de la cotidianeidad y desde la intimidad del yo poético. Según el enfoque lingüístico-cognitivo de George Lakoff y Mark Johnson (2009), las metáforas están presentes en la vida cotidiana, no solo a través del lenguaje — cuya elaboración en la poesía es más compleja que en el registro oral-, sino también en el pensamiento y la acción. Para dichos 
teóricos, nuestros pensamientos y actos son fundamentalmente de naturaleza metafórica:

Para la mayoría de la gente, la metáfora es un recurso de la imaginación poética y los ademanes retóricos, una cuestión de lenguaje extraordinario más que ordinario. Es más, la metáfora se contempla característicamente como un rasgo sólo del lenguaje, cosa de palabras más que pensamiento acción. Nosotros hemos llegado a la conclusión de que la metáfora, por el contrario, impregna la vida cotidiana, no solamente el lenguaje, sino también el pensamiento y la acción (Lakoff y Johson, 2009, p. 39).

A partir de ello, ambos investigadores sostienen que el pensamiento humano es, en gran medida, metafórico. Las metáforas se presentan en el lenguaje cotidiano formando una red compleja e interrelacionada con otras ideas. No solo es una operación del lenguaje, pues para su construcción se toma como base la vida cotidiana. Las consideraciones de Lakoff y Johnson resultan pertinentes, en tanto ambos analizan la metáfora más allá de un enfoque retórico, cuyo fundamento es la construcción conceptual que realiza una persona que no solamente toma como referencia a la palabra escrita. "Las metáforas como expresiones lingüísticas son posibles, precisamente, porque son metáforas en el sistema conceptual de una persona” (p. 42), afirman.

Aunque puede interpretarse como un deslinde del texto poético, lo cotidiano nutre a la creación lírica, pues el poeta escribe no solamente a partir de su mundo interior sino también desde lo que acontece en su entorno inmediato. En todo caso, la mirada de lo cotidiano es siempre un punto de partida sobre el cual se apoya el yo poético en Viviendo el tiempo.

En su propuesta, Lakoff y Johnson distinguen tres tipos de metáforas: las estructurales, las orientacionales y las ontológicas. Las metáforas estructurales son aquellas en las que un concepto está estructurado en términos de otro. Por ejemplo, cuando decimos que el tiempo es dinero. Las metáforas orientacionales organizan un sistema global de conceptos con relación con otro. La mayoría de ellas tienen que ver con orientación espacial: arriba-abajo, adentro-afuera, etc. Por ejemplo, cuando decimos: "eso me levantó el ánimo" o "caí en una depresión”. Finalmente, las metáforas ontológicas conciernen en gran parte a la poesía, puesto que "[las] utilizamos para entender acontecimientos, acciones, actividades y estados" (p. 69), y se las considera como si fueran entidades y sustancias. Por ejemplo, cuando decimos que la mente es una máquina: "voy a perder el control” o "mi 
cerebro no funciona hoy". Suelen darle a lo abstracto una dimensión corpórea. El dolor no se puede cuantificar, pero a través de la metaforización se le otorga una cualidad concreta. En el poemario citado de Yolanda Westphalen, el tiempo, que es un elemento de naturaleza abstracta, es representado como si fuera un ser vivo.

En Viviendo el tiempo la metáfora principal está relacionada con el tiempo que condiciona los estados anímicos del yo lírico. A partir de nuestro análisis determinaremos de qué manera se aplica la metaforización. De allí que la propuesta de la lingüística cognitiva resulte idónea, pues no solo identifica a la metáfora, sino que señala su conceptualización. Este enfoque no es nuevo para los estudios literarios, puesto que a partir de él se han analizado las poéticas y los mecanismos de producción textual de obras literarias, más precisamente poéticas. Por citar un caso, tenemos al reconocido investigador Camilo Fernández Cozman, quien ha utilizado el enfoque de Lakoff y Johnson para estudiar la poesía de Blanca Varela y de Carlos López Degregori.

Stefano Arduini (2000), destacado investigador italiano, ponderó los alcances de este enfoque lingüístico-cognitivo para el estudio de la metáfora y el análisis poético:

Desde este punto de vista, pues, las figuras, y la metáfora en particular, representan algo más importante que un simple ornamento o un mero desvío de un nivel neutro que debe ser necesariamente reconstruido. Con Lakoff y Johnson, la metáfora es un medio esencial para leer el mundo (Arduini, 2000, p. 25).

Es decir, la metáfora no solamente embellece al poema, sino también encierra un sentido que parte de la mirada del yo lírico hacia su entorno.

La creación poética, como afirma Enrique Huelva Unterbäumen (2019), en relación con el tiempo, es también un proceso de creación metafórica:

En otras palabras: la poesía y el pensamiento filosófico conceptualizan metafóricamente la temporalidad en procesos que tienen como dominio-meta la experiencia de la temporalidad (proto-temporalidad) y como dominio-fuente las metáforas generales (Huelva, 2019, p. 94).

Llevado al terreno de la creación poética, la metaforización del tiempo parte de una experiencia que incluye, a su vez, una vivencia o emoción determinadas. $\mathrm{Al}$ analizar los poemas encontraremos, efectivamente, tal estructuración de conceptos. 
La metáfora es una figura recurrente en la poesía y Viviendo el tiempo no es la excepción. De allí que nuestro análisis de carácter hermenéutico se centre en esta figura, pero no solo para identificarla, sino también para conocer cómo se presenta la conceptualización del tiempo en el poema.

Para Arduini la metáfora "conserva el primado entre los tropos" y "actualiza operaciones que comprometen procesos mentales" (p. 80). Asimismo, sostiene que esta figura "constituye una versión del mundo y determinan su pensabilidad” (loc. cit.). En ese sentido, la metáfora implica una visión personal de la realidad. El yo poético configura así la representación de su vida personal, social y cultural. Dado que un texto poético responde a una concepción personal, se enmarca dentro de un campo retórico determinado. En ese sentido, las figuras son modalidades o formas de pensamiento que nos permiten conocer expresivamente la visión del yo lírico en un determinado poema. La metáfora es una de las figuras más estudiadas y, en los poemas de Viviendo el tiempo, encontramos una relación entre la metáfora del tiempo asociada a emociones particulares del yo poético.

Asimismo, como sostiene Carlos García-Bedoya (2019), la metáfora es un tropo que "procede por semejanza. Se sustituye una expresión por otra que transmite un sentido análogo” (p. 159). Esta es una definición clásica, pero que no excluye la idea de conceptualización de un elemento, que en el caso de Lakoff y Johnson es más amplia. En su análisis del campo retórico, Arduini afirma que entre mundo y lenguaje hay una estrecha relación a la que ha llamado de interacción o de circularidad. "Es importante subrayar que esta relación no aparece solo a través de la expresión cada vez que hablamos, es en la expresión donde se realiza la unidad mundo-lenguaje" (Arduini, 2000, p. 45).

Esta representación del mundo, efectivamente, configura una visión del yo poético, la cual está representada a partir de las metáforas como figura principal en el poemario Viviendo el tiempo. El análisis de Arduini nos permite detectar este mecanismo y cómo se expresa en cada uno de los poemas. En ese sentido, las figuras son modalidades o formas de pensamiento que nos permiten conocer expresivamente la visión del yo lírico en un determinado texto poético. La metáfora es una de las figuras más estudiadas y, en los poemas de Viviendo el tiempo, encontramos una relación entre la metáfora del tiempo relacionada con emociones particulares del yo poético. 


\section{Cinco poemas de Viviendo el tiempo: análisis}

Viviendo el tiempo (2007) es el noveno poemario de Yolanda Westphalen y, como señalamos anteriormente, es donde mejor se aprecia la participación del tiempo como tema central de los poemas. Este libro se encuentra conformado por 47 textos de breve extensión que no llevan título, pero están enumerados de manera consecutiva. Algunos poemas son de apenas dos versos, mientras que otros como máximo tienen 12. Esta brevedad se evidencia, a su vez, en cada verso que consta de entre 4 y 11 sílabas. Dicha concisión le otorga profundidad al poema, que es una marca en la poética de Westphalen. La investigadora Yolanda Westphalen Rodríguez señala sobre el título y estructura general de los poemas del libro:

El uso del gerundio ("viviendo"), esa forma verbal que expresa la simultaneidad de la acción con el tiempo en que se habla, establece para la autora la coincidencia de la acción poética con el tiempo, poesía que no solo vive y captura el tiempo, sino que lo trasciende (Westphalen, 2018, p. 27).

El eje temático central del poemario es el tiempo y el dominio que ejerce este sobre el yo poético. Este, como indica la investigadora Westphalen Rodríguez, es un agente que marca la acción y que también deja huella en el sujeto lírico que busca la trascendencia a través de las palabras. Esta relación de dominio se configura desde la mirada de lo cotidiano. El paso de los días se refleja en los cambios de estación y en los fenómenos atmosféricos como la lluvia, que es una constante a lo largo del poemario. Las sensaciones de dolor, soledad y tristeza marcan el tono de los poemas que transmiten la angustia del yo poético por el paso del tiempo. Para el sujeto lírico, sin embargo, solo a través de las palabras hay una posibilidad de trascendencia.

Para nuestro análisis hemos seleccionado cinco poemas representativos para evidenciar cómo se presenta la metaforización del tiempo.

Empezaremos con el poema 6:

Desmenuzo el perfil 1

de una palabra 2

y crece entre 3 
la tiniebla de lo oscuro4

el acoso $\quad 5$

voraz del tiempo. 6

Aquí palabra y tiempo se confrontan en una batalla simbólica en la que este último se impone. Como indican los versos 5 y 6, el tiempo acosa y es voraz. Esta corporeización del tiempo es una representación metafórica de acuerdo con el esquema de Lakoff y Johnson. El tiempo es un ente abstracto que cobra una dimensión actuante bajo la forma de una metáfora del tipo ontológica. El yo poético se encuentra a expensas del tiempo, que está siempre al acecho y ejerce su poderío.

Veamos a continuación el poema 11, donde el yo poético expresa una emoción a partir de su relación con el tiempo:

11

¡Qué triste envejecer 1

al borde de la vida! 2

Las horas marcan 3

tu piel 4

Cada arruga $\quad 5$

es un grito de protesta 6

es un árbol sin raíz $\quad 7$

es un bosque huérfano. $\quad 8$

Entre el vivir y el morir 9

¡Qué lento el paso! 10 
Este poema, a diferencia del anterior, no solo nos muestra la relación sujeto-tiempo, sino que además expresa una emoción del yo poético. La angustia que suscita el paso del tiempo y la acción que ejerce aquel en su cuerpo: véanse los versos 3 y 4 . Asimismo, se produce una reacción, “un grito de protesta” y el remate del poema reconecta con los versos 1 y 2 : el inicio y el fin que se anuncia en el último tramo ("borde") de la existencia. Aquí también la metaforización del tiempo se manifiesta en los versos "las horas marcan / tu piel".

En el poema 24 la metaforización es mucho más directa: "el tiempo es actualidad dinámica" y "El tiempo es la rueda" (versos 1 y 3). Veamos el poema completo:

\section{4}

El tiempo es actualidad dinámica $\quad 1$

Es proximidad y lejanía

El tiempo es la rueda

inoxidable y perenne

de la vida.

La metaforización de tipo ontológica nos muestra al tiempo como una rueda “inoxidable y perenne". A diferencia de los dos poemas anteriores aquí no se expresan los sentimientos o emociones del yo poético, sino más bien nos muestra cómo actúa el tiempo.

A continuación, analicemos el poema " 26 ":

Viviendo el tiempo

palpas la soledad

Y en la oscuridad de lo oscuro se enciende el silencio. 
El gerundio nos muestra la simultaneidad del vivir con el sentir. Por un lado, se vive el tiempo y, a través de este, "palpas la soledad". Los versos 3 y 4 rematan esta sensación de angustia y desolación representados, por un lado, a través de la oscuridad y el silencio. La metaforización del tiempo aquí es menos directa, pero si nos ceñimos al esquema de Lakoff y Johnson, veremos que este elemento se evidencia en su dimensionalidad. El tiempo opera como un camino o derrotero solitario. El yo lírico expresa su melancolía por esta revelación.

Veamos ahora otro poema donde el tiempo vuelve a ejercer su dominio sobre el yo poético y este lo confronta a través de la creación literaria:

\section{6}

Y el tiempo me exige 1

que viva el esplendor 2

de la palabra 3

en fosforescente

4

devenir

5

Siempre transfigurada.

6

A diferencia del poema " 6 ", donde la palabra es un refugio para hacerle frente al tiempo, aquí este más bien impulsa al yo poético a "que viva el esplendor / de la palabra”. Además, se advierte que el acto creativo varía en intensidad y es cambiante: "siempre transfigurada". La relación que se establece entre el yo poético y el tiempo es mucho más visible tal como muestra el primer verso: "Y el tiempo me exige".

\section{Conclusiones}

1. En la poesía de Yolanda Westphalen el tiempo es un elemento que permite a la voz poética reflexionar sobre el sentido de la existencia. En ese sentido, la obra de dicha autora es singular respecto a sus pares de la generación del 50 e, incluso, de la generación del 60, donde se le adscribe. 
2. De acuerdo con la propuesta de Lakoff y Johnson, la metaforización del tiempo en el poemario Viviendo el tiempo se presenta otorgándole una dimensión corpórea a un ente abstracto. Es así que el tiempo ejerce una acción física y emocional sobre el sujeto lírico. Esta configuración se presenta a lo largo del libro, tal como se aprecia en los cinco poemas seleccionados para este análisis.

3. La mirada del entorno, es decir del mundo cotidiano, está condicionada por el tiempo. Este elemento se manifiesta a través de la sucesión de días, del cambio de estación o de fenómenos atmosféricos como la lluvia. Estos escenarios son los puntos de partida para la construcción de cada poema.

4. La palabra, entendida como el ejercicio poético, es un mecanismo de resistencia frente al dominio que establece el tiempo sobre el sujeto lírico. A través de esta acción se busca trascender la existencia que en algunos casos se percibe breve, en otras como un devenir incierto y lento. Estas percepciones están condicionadas por un estado anímico particular, que a su vez determina la manera en que el tiempo es representado metafóricamente a lo largo del poemario.

\section{Nota}

1 En 1999, la volvió a incluir en el tomo I de la antología Poesía peruana. Siglo XX, editada por Ediciones Copé.

\section{Contribución del autor}

Jaime Cabrera Junco ha participado en la elaboración, la compilación de datos, la redacción y el consentimiento de la versión final del presente artículo.

\section{Fuente de financiamiento}

La investigación es autofinanciada.

\section{Conflictos de interés}

Ninguno.

\section{Trayectoria académica}

Jaime Cabrera Junco es bachiller en Comunicación Social y maestrista de la maestría de Escritura Creativa de la Universidad Nacional Mayor de San 
Marcos. Autor del poemario El cantar de las agujas (2020, inédito) y gestor cultural del proyecto literario Lee por gusto. Artículos, entrevistas y reseñas de libros de su autoría han sido publicados en los diarios El Comercio y Perú 21 y la revista Buensalvaje.

\section{Referencias bibliográficas}

Arduini, S. (2000). Prolegómenos para una teoría general de las figuras. Universidad de Murcia.

Cachay, C. (2020). La deshumanización en Objetos enajenados (1971) de Yolanda Westphalen. Metáfora, 3(5). http://www.metaforarevista.com/index.php/ meta/article/view/60/61

Carrillo, F. (1965). Antología de la poesía peruana joven. La Rama Florida y Biblioteca Universitaria.

Castañeda, E. (1995). Aproximación a una antología. En C. Barcellos de Zarria (coord.), Antología poética: peruanas del siglo XX (III-VIII). Ediciones G.A.P.

Cevallos, L. (1967). Los nuevos. Universitaria.

Chirinos, E. (2019). La poesía peruana en los años sesenta. En G. Pollarolo y L. Chueca (eds.). Historia de las literaturas en el Perú. Volumen 4. Poesía peruana: entre la fundación de su modernidad y finales del siglo XX (269-296). Fondo Editorial de la Pontificia Universidad Católica del Perú y Casa de la Literatura Peruana.

Forgues, R. (2004). Yolanda Westphalen y Carmen Luz Bejarano. La mirada existencial. En R. Forgues (ed.), Plumas de Afrodita. Una mirada a la poeta peruana del siglo XX (91-102). Editorial San Marcos.

García-Bedoya, C. (2019). Hermenéutica literaria. Una introducción al análisis de textos narrativos y poéticos. Cátedra Vallejo.

Huelva, E. (2019). En los vértices del tiempo. Metáforas conceptuales del tiempo y sus variaciones en la poesía y el pensamiento filosófico. Cultura, Lenguaje y Representación. Revista de Estudios Culturales de la Universitat Jaume I (XXII), 75-97. http://www.e-revistes.uji.es/index.php/clr/article/view/3786

Lakoff, G. y Johnson, M. (2009). Metáforas de la vida cotidiana. Cátedra.

Salvador, G. (2020). Visión del lenguaje en un poema de Universo en exilio (1984) de Yolanda Westphalen: análisis retórico de "6". Metáfora, 3(5). http://www. metaforarevista.com/index.php/meta/article/view/62/63 
Troiano, M. (2008). “Viviendo el tiempo" de Yolanda Westphalen: una elegía para perdurar. https://scriptura-blog.blogspot.com/2008/12/viviendo-el-tiempo-de-yolanda.html

Westphalen, Y. (2018 a). Obra completa. Poesía. Fondo Editorial de la Universidad Nacional Mayor de San Marcos.

Westphalen, Y. (2018b). Obra completa. Cuentos, ensayos y artículos periodísticos. Fondo Editorial de la Universidad Nacional Mayor de San Marcos.

Zapata, M. A. (2002). Moradas de la voz: notas sobre la poesía hispanoamericana contemporánea. Facultad de Letras y Ciencias Humanas de la Universidad Nacional Mayor de San Marcos e Instituto de Investigaciones Humanísticas. 